\title{
Scientific-technical investigations for development of optical multifunctional components for deflection, splitting and shaping of light beams
}

\author{
Sebastian Henkel ${ }^{1, *}$, Andrea Barz ${ }^{1}$, Jens Bliedtner ${ }^{1}$ and Kurt Kleinen ${ }^{2}$ \\ ${ }^{1}$ Ernst-Abbe University of Applied Sciences, Carl-Zeiss-Promenade 2, 07745 Jena, Germany \\ ${ }^{2}$ MICROS Optics GmbH \& Co. KG, Taubenbacher Weg 80, 07318 Saalfeld, Germany
}

\begin{abstract}
Many optical applications require splitting or deflection of light beams. This usually is achieved by usage of standard plane optical elements such as mirror systems or prismatic elements. However, these components are usually limited to one certain function and the manufacturing process for achieving precise functionality is very demanding. The presented research topic shows the development of new optical multifunctional components that introduce certain advantages compared to existing plane optical components. The basic roof-element configuration is based on glass cuboids joined together under a certain angle that can be fine adjusted during the manufacturing process. The components are therefore rather simply built and robust, and can fulfil different optical functions simultaneously by modifications of the glass cuboids. A process chain was developed for efficient and flexible manufacturing of such plane optical elements. Technical investigations were made on novel manufacturing processes such as ultrasonic assisted grinding and ultrafine grinding for fabrication of the cuboid parts, as well as diffusion joining being one possibility to create the basic roof-element shape.
\end{abstract}

\section{Introduction}

Optics designers can choose from a broad variety of plane optical elements. Reflective prisms for example exist in many different geometries to achieve all kinds of beam or image splitting, as well as image inversion in optical systems, such as telescopes, cameras or digital projectors. In contrast to spherical optics, plane optics are not used for optical magnification or optical reduction of an image. Instead, these components are used to change the direction of light beams, for example to change the orientation and position of an image or to achieve a parallel offset. A beam path can be advantageously "folded" with these elements within an optical system to reduce its overall length. Beam splitting components, such as splitter plates or splitter cubes, in turn, serve to split a beam into several partial beams. When it comes to functionality all existing parts suffer from the fact, that they can be usually used only for image inversion or beam splitting or light deflection. So, they are not multifunctional and often demand high manufacturing efforts. Due to this a new development of innovative adjustable roof elements, which can fulfil various optical functions simultaneously and are of simple and robust design, was sought. These new multifunctional elements can be used for beam splitting and guidance as well as for beam focusing or expansion of laser and light beams. Their application is also conceivable in measuring systems or laser material processing machines.

\section{Basic configuration and possible variations of new multifunctional components}

The basic configuration, a so-called roof element, consists of two glass cuboids joined together under an angle of $90^{\circ}$ either by an adhesive or by the process of diffusion joining. The cuboids can be coated with arbitrary metal or dielectric coatings chosen for the desired application. What makes the element unique is the possibility of fine adjusting the angle between the cuboids in the range of a few arc seconds during or after the joining process by inserting a small wedge-like element between the cuboids as seen in fig. 1. Therefore, less precision in angle manufacturing is needed, since the element can be fine adjusted afterwards. This is applied for patent [1].
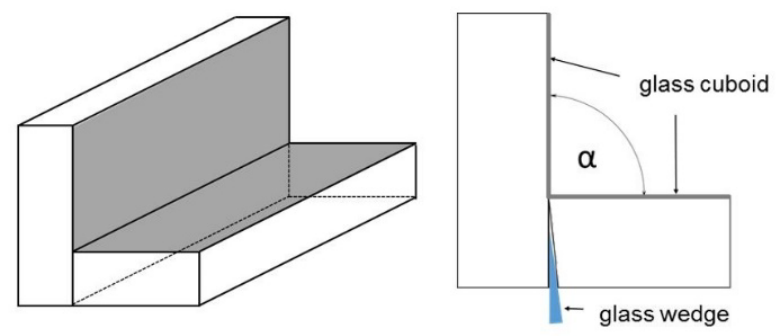

Fig. 1. Schematic view on adjustable roof-element: 3D illustration of basic set-up (left); joining and simultaneous angle adjustment by wedge positioning (right)

\footnotetext{
$\overline{\text { * Corresponding author: sebastian.henkel@eah-jena.de }}$
} 
A high modularity and flexibility can be achieved by variations of the basic configuration. A usage as a multiple beam-splitter is possible using partially transmissive coatings (fig. 2 left). Altering the shape of the glass cuboids (e.g. adding spherical or aspherical surfaces) offers the chance of focussing or shaping the incident light beams in addition to a beam split (fig. 2 right).
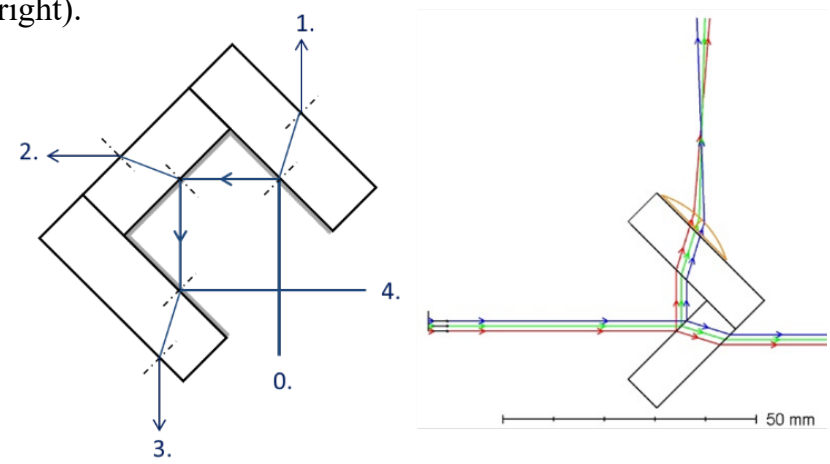

Fig. 2. Examples of component modifications for multiple beam splitting and beam shaping

Further ideas include the addition of a freeform surface or a microstructure on a cuboid surface as well as usage of $\lambda / 4$ - coatings for changing the polarisation conditions of light rays.

\section{Novel process chain for component manufacturing}

The presented project also includes investigations on novel manufacturing technologies to offer an improved process chain for component production. It is aimed to replace or reduce lapping or polishing steps, especially for more complex surfaces, which would be normally used in optics manufacturing and are rather time consuming. This can be achieved by implementing the technologies ultrasonic assisted (US) grinding, ultrafine grinding and a more deterministic polishing process using NC-controlled machines and kinematic simulations for uniform material removal. Fig. 3 shows a potential process chain.

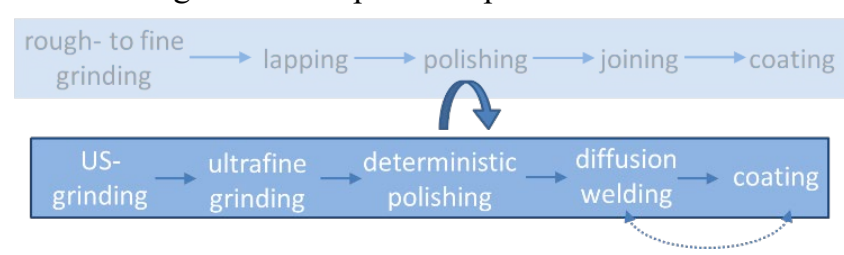

Fig. 3. Conventional process chain (above) and novel process chain (below) for optics manufacturing

US grinding can be done on a 5-axis grinding machine, offering high shape flexibility. This technology is characterized by a high frequency, longitudinal oscillation of the tool in addition to its rotation. This kinematic superimposition results in higher cutting speed [2] and reduction of process forces up to over $40 \%$. Own investigations and literature have shown the tendency to decrease sub surface damages. The application of an ultrafine grinding process afterwards on the same 5-axis machine can strongly reduce the surface roughness of the glass components. Special resin bond tools from company GÜNTER EFFGEN GMBH are used to achieve roughness values down to $R q=10 \mathrm{~nm}$ on fused silica. This shortens the time for an afterwards polishing process over $50 \%$ compared to polishing a fine lapped surface, regarding a polishing roughness goal of $R a=5.5 \mathrm{~nm}$ (tactile measurement). A further innovative technology of the process chain is diffusion joining. Applying pressure and heat to the joined elements enables a material bond with a strength comparable to the bulk material of the glass.

\section{Results and summary}

Next to the preparation of the glass cuboids and the process investigations, a set-up for mounting the elements and carrying out the angle fine-adjustment was constructed and fabricated. The mounting device with the clamped glass cuboids was inserted into the beam path of a goniometer. Using suitable fine adjustment screws, the internal angle of the roof-element can be successively changed via a freely movable aluminium leg and measured inline until the desired angle is reached. The wedge is then inserted to keep the position stable and the joint is fixated by UV-curing an adhesive film. This setup enables a high precision angle adjustment with deviations to about 3 arc seconds. During curing of the adhesive, the deviations will increase but still stay below 20 arc seconds. This corresponds to the specified requirements of $90^{\circ}$ prisms for higher demands. [3][4]

The investigations have shown, that it is possible to build novel multifunctional optical elements with fine adjustable angle precision based on a simple and costeffective basic roof-element configuration. Different functions of light deflection, beam splitting, beam shaping and more can be achieved, which was also proofed with some simple demonstrators. Furthermore, technological investigations on a novel process chain for manufacturing such elements were carried out. Based on the basic roofelement design it is possible to create inexpensive, lightweight and monolithic optical elements flexibly adaptable to many different required applications.

The authors gratefully acknowledge financial support by the German Federal Ministry of Economy and Energy in the funding program ZIM (funding reference: ZF4039608DF7).

\section{References}

1. EP 3528027 A1: Optical assembly for changing the direction of light beams with at least two mirror parts. (2019)

2. C. Schopf, R. Rascher, P. Sperber: Vor- und Nachteile der Ultraschalltechnologie beim Schleifen von optischen Flächen. Future Glass - from the Tradition to the High-Tech-Product, Focus: Optical Glass, Zwiesel. (2010)

3. https://www.thorlabs.de/newgrouppage9.cfm?object group_id=142; viewed on 24.03.2020.

4. https://www.thorlabs.de/newgrouppage9.cfm?object group_id=142; viewed on 24.03.2020. 